\title{
Speckle reduction of endovascular optical coherence tomography using a generalized divergence measure
}

\author{
Kyle H. Y. Cheng, ${ }^{1,2}$ Edmund Y. Lam, ${ }^{3}$ Beau A. Standish, ${ }^{1,4}$ and Victor X. D. Yang, ${ }^{1,2,4,5, *}$ \\ ${ }^{1}$ Biophotonics and Bioengineering Laboratory, Ryerson University, 350 Victoria Street, Toronto, Ontario M5B 2K3, Canada \\ ${ }^{2}$ The Edward S. Rogers Sr. Department of Electrical and Computer Engineering, University of Toronto, \\ 10 King’s College Road, Toronto, Ontario M5S 3G4, Canada \\ ${ }^{3}$ Department of Electrical and Electronic Engineering, The University of Hong Kong, Pokfulam Road, Hong Kong, China \\ ${ }^{4}$ Department of Electrical and Computer Engineering, Ryerson University, 350 Victoria Street, Toronto, Ontario M5B 2K3, Canada \\ ${ }^{5}$ Department of Medical Imaging, St. Michael's Hospital, 30 Bond Street, Toronto, Ontario M5B 1W8, Canada \\ *Corresponding author: yangv@ee.ryerson.ca
}

Received March 28, 2012; revised May 25, 2012; accepted May 29, 2012;

posted May 30, 2012 (Doc. ID 165624); published July 11, 2012

\begin{abstract}
Endovascular optical coherence tomography (EV-OCT) is an emerging intravascular imaging technique for observing blood vessel walls. Fluctuating speckle noise, especially during rapid pull-back, can severely degrade the visibility of morphological structures. Moreover, the speckle pattern varies in different parts of the image due to beam divergence and is further complicated by interpolation through the coordinate transformation necessary for displaying the rotary scanning images, challenging the use of frequency domain analysis. In this study, a computationally efficient method using a generalized divergence regularization procedure is presented to suppress speckle noise in EV-OCT images. Results show substantial smoothing of the grainy appearance and enhanced visualization of deeper structures as demonstrated in porcine carotid arteries. (c) 2012 Optical Society of America

OCIS codes: $170.1610,170.4500,100.2000,100.2980,100.3190$.
\end{abstract}

Endovascular optical coherence tomography (EV-OCT) is an emerging intravascular imaging technique for major blood vessel wall visualization with $\sim 15 \mu \mathrm{m}$ resolution [1]. Numerous studies have demonstrated that EV-OCT can distinguish among the media, external elastic lamina, and the adventitia clearly in normal arteries [1]. In addition, EV-OCT has also been shown to delineate the fibrous cap, lipid deposits, and neovascularization in atherosclerotic plaques [1]. However, due to water absorption and tissue scattering, the penetration depth of EV-OCT is limited to $\sim 2 \mathrm{~mm}$. The presence of speckle noise further complicates visualization as its modulated appearance can further distort the faint morphological signals of deeply situated structures (e.g., adventitia). In between B-mode frames of an EV-OCT pullback image sequence, the speckle pattern fluctuates dramatically due to motion artifacts from various sources $[2, \underline{3}]$.

Common hardware based approaches include frequency [4] and angular compounding [5]. They are robust ways of speckle suppression as speckle properties vary across wavelengths or different illumination angles [6]. However, since they require additional components, they may not be applicable to existing commercial EV-OCT systems.

Various studies have been devoted to speckle reduction using digital image processing techniques. Many of these are based on integral transforms and the manipulation of the associated coefficients [7-9]. Others rely on various algebraic iterations $[10,11]$. Optical beam divergence, nonuniform spatial sampling of the rotary scanning mechanism, and the subsequent interpolation all contribute to the speckle pattern shape and orientation variation in different parts of the image. These represent challenges to coefficient based speckle reduction methods. Since a typical EV-OCT pullback image sequence consists of several hundred images, algorithms with complex algebraic operations may not be efficient to tackle the large amounts of data.

In this Letter, we propose a simple, fast converging iterative algorithm modified from [12]. It is demonstrated that by regularizing the image during each iteration using a generalized divergence measure, $\beta$-divergence, speckle suppression and edge preservation can be achieved simultaneously. Moreover, one can freely adjust the tradeoff between speckle suppression and edge preservation by adjusting a single parameter.

$\beta$-divergence was used for blind source separation [13] and is defined as

$$
d_{\beta}(x, u)=\frac{1}{\beta(\beta-1)}\left[x^{\beta}+(\beta-1) u^{\beta}-\beta x u^{\beta-1}\right],
$$

where $x$ equals, in our case, the despeckled image, and $u$ denotes a reference image. In this equation, $\beta$ is a scalar parameter to be chosen. The $\beta$-divergence has the important property, where only when $x=u$ will the divergence measure vanish. It does not subject the image to error by generating undefined pixels since there are no reciprocal or logarithmic terms of $x$ or $u$ for $\beta>1$. The measure was also shown to be robust against outliers [13].

In this study, the $\beta$-divergence algorithm was developed to first enforce the least-square consistency of the despeckled image with the measured data, and then focus on the matching of details between the image and a reference image $u$ using the $\beta$-divergence regularization measure. To achieve this, the following inverse problem was solved:

$$
\begin{aligned}
\hat{x}= & \underset{x}{\arg \min }\|y-P x\|^{2} \\
& +\lambda \sum_{i} \frac{1}{\beta(\beta-1)}\left[x_{i}^{2 \beta}+(\beta-1) u_{i}^{2 \beta}-\beta x_{i}^{2} u_{i}^{2(\beta-1)}\right],
\end{aligned}
$$



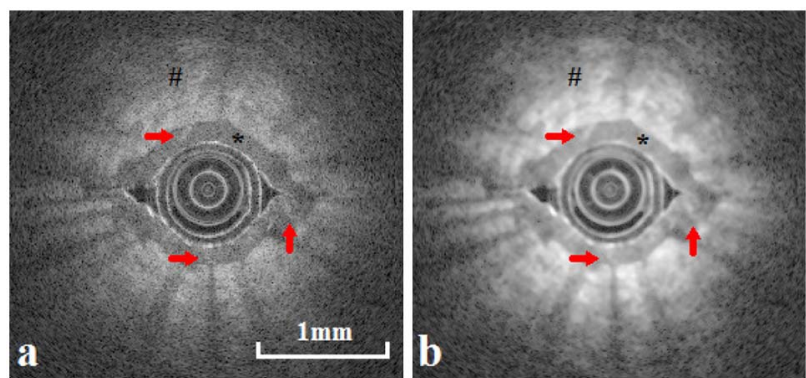

Fig. 1. (Color online) (a) Original finger skin image; (b) despeckled finger skin image. (*) indicates the stratus corneum, (\#) indicates the dermis, and the red arrows point to the sweat glands.

where $y$ is the measured image, $P$ is a downscaled point spread function, and the subscript $i$ indicates the $i$ th pixel of the image. By taking the derivative of Eq. (2) with respect to $x$, we arrive at the iterative scheme:

$$
\begin{aligned}
\hat{x}_{i}^{(n+1)}= & \hat{x}_{i}^{n}-\varepsilon\left\{2\left(P^{*} P x-P^{*} y\right)_{i}+\frac{\lambda}{\beta(\beta-1)}\right. \\
& \left.\times\left[2 \beta\left|x_{i}\right|^{2 \beta-1}-2 \beta\left|x_{i}\right|\left|u_{i}\right|^{2(\beta-1)}\right]\right\},
\end{aligned}
$$

where $P^{*}$ is the adjoint of $P, \varepsilon$ is the iteration constant, and $\lambda$ is the regularization constant. The image was normalized at each iteration to limit its bandwidth.

OCT images were obtained using a commercial Lightlab C7-XR Fourier Domain OCT system (Lightlab Imaging). To demonstrate the speckle suppression effect of the algorithm, an initial test image of human finger skin was obtained by pressing two fingertips against the imaging catheter tip. In vivo endovascular OCT imaging of the porcine carotid arterial wall image was obtained subsequently with eccentric placement of the imaging catheter to demonstrate the versatility of our algorithm to suppress speckle at different parts of the image. The details of animal imaging protocol have been previously described elsewhere [14]. All animal procedures were approved by St. Michael's Hospital (Toronto, Ontatio) Animal Care Committee.

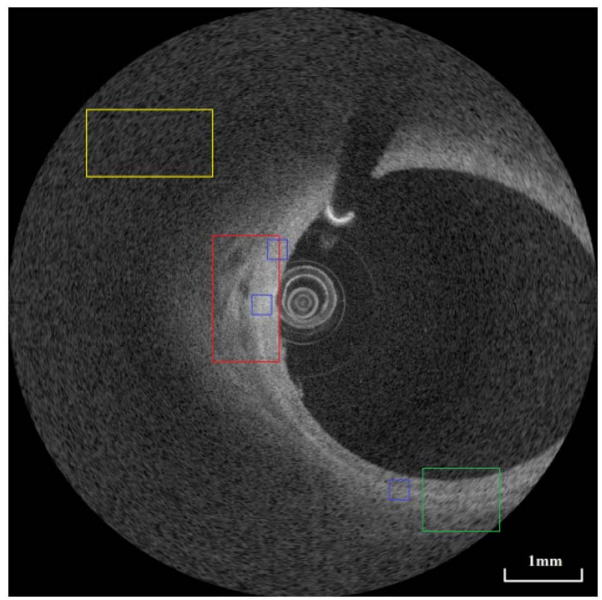

Fig. 2. (Color online) Original porcine carotid artery EV-OCT image. The red ROI indicates the signal region and the yellow ROI indicates the noise region used in the metrics calculation. The green ROI indicates the zoomed region in Fig. 5. The three blue ROIS are used for ENL calculations.

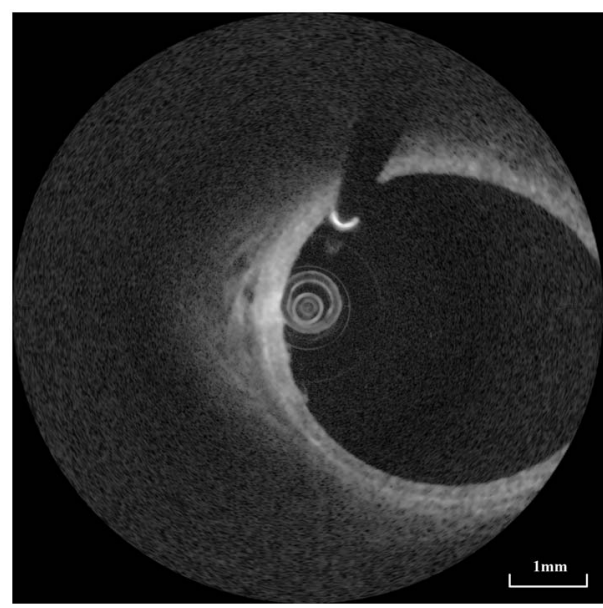

Fig. 3. Despeckled porcine carotid artery EV-OCT image.

In the algorithm, $\varepsilon$ was chosen to be 0.75 and $\lambda$ was set to 0.6 determined interactively by the OCT operator. The reference image $u$ was generated by passing the unprocessed image to a $12 \times 12$ median filter and then filtered by a $5 \times 5$ averaging filter. Median filters have been used before for speckle suppression [15], while averaging filters can effectively remove additive noise, since speckle in the EV-OCT image after logarithmic compression becomes additive. The algorithm was set to run for 40 iterations as the result started to converge to $\sim 0.002$ mean square difference from $u$.

Figure 1(a) shows the original finger skin image, and Fig. 1(b) demonstrates the despeckled finger skin image processed with our proposed algorithm. Most of the features were preserved in the despeckled image due to the robustness of $\beta$-divergence against outliers, while the grainy appearance was mostly smoothed. Figures 2 and 3 show the original and despeckled $(\beta=3)$ carotid arterial wall image, respectively. After speckle suppression, the grainy appearance of the tissue was clearly removed.

To quantitatively evaluate the performance of the algorithm on real endovascular images, a number of metrics were calculated for a region of interest (ROI) encompassing high signal regions (media) and low signal regions (adventitia) versus a noise background as depicted in Fig. 2. The signal-to-noise ratio (SNR) was defined as $\mathrm{SNR}^{-}=20 \log \left(x_{\operatorname{lin}} / \sigma_{\operatorname{lin}}\right)$, where $x_{\text {lin }}$ is the maximum intensity in the ROI in linear scale. $\sigma_{\text {lin }}$ is the standard
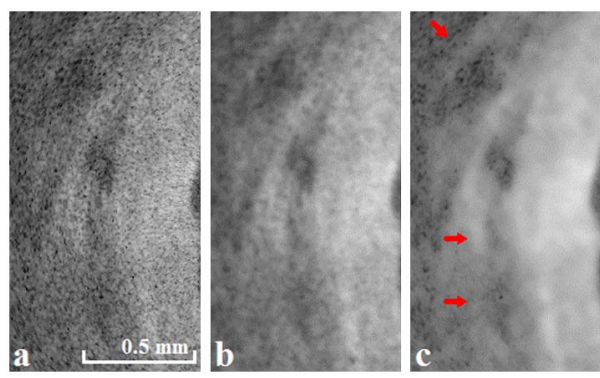

Fig. 4. (Color online) (a) Enlarged view of the red ROI in the original porcine arterial wall image; (b) enlarged view of the red signal ROI in the despeckled image processed by I-divergence algorithm; (c) enlarged view of the red signal ROI in the despeckled image processed by our proposed algorithm. The arrows indicate structures that are much less visible in the unprocessed image. 

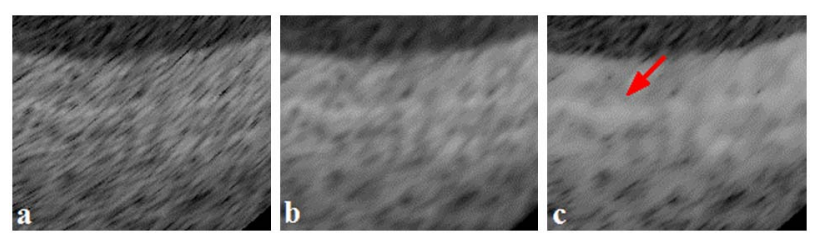

Fig. 5. (Color online) (a) Enlarged view of the green ROI in the original image; (b) enlarged view of the green signal ROI in the despeckled image processed by I-divergence algorithm; (c) enlarged view of the green signal ROI in the despeckled image processed by our proposed algorithm. The red arrow indicates the clearer visualization of the external elastic lamina.

deviation of the noise region in linear scale. The contrastto-noise ratio (CNR) was defined as $\mathrm{CNR}=10 \mathrm{log}$ $\left[\left(\mu_{x}-\mu_{b}\right)\left(\sigma_{x}^{2}-\sigma_{b}^{2}\right)^{0.5}\right]$, where $\mu$ and $\sigma$ are the mean and standard deviation and subscripts $x$ and $b$ denote the $\mathrm{ROI}$ and noise region, respectively. The equivalent number of looks (ENL) was defined as ENL $=\mu_{x}^{2} / \sigma_{x}^{2}$ and is averaged between three ROIs depicted in Fig. 2. An edge preservation parameter is defined previously [흐]. Briefly, the larger the parameter, the more edges are preserved. Figures $\underline{4(\mathrm{a})}, \underline{4(\mathrm{~b})}$, and $\underline{4(\mathrm{c})}$ show the enlarged views of the ROIs without processing, processed by [12] and processed by our proposed algorithm, respectively. As noted by the arrows in Fig. 4(c), visually these structures are very difficult to be discerned in Fig. $\underline{4(\mathrm{a})}$. Figure $\underline{5}$ shows a region far from the catheter without and with speckle suppression. The result demonstrates that our algorithm can better delineate features, despite the fact that they are located further from the imaging catheter where a diverging optical beam provides less SNR. This also demonstrates our technique is robust against variations in speckle orientation.

Table 1 shows the results when $\beta=1.5,3$, and 4 . In all cases, the SNR, CNR, and ENL all showed improvement compared to the original image. The result obtained by a $12 \times 12$ median filter was also listed. It produces very good SNR, but edges were poorly preserved. To further show how $\beta$ affects the speckle suppression effect, CNR and edge preservation were plotted against $\beta$ in Fig. $\underline{6}$. When $\beta$ is less than 3 , the CNR is higher but the edge preservation drops, and vice versa when $\beta$ is larger than 3 . Thus, $\beta$ acts as an adjustable parameter that controls the trade-off between speckle suppression and edge preservation. Such trade-off adjustment is needed when one wants to see different features. For example, when one wants to evaluate large area atherosclerotic plaques, $\beta$ should be set lower than 3 to apply stronger speckle suppression so as to clearly delineate different parts of the plaque. However, when one wants to seek neovascularization, $\beta$ should be set higher than 3 in order to preserve the vessel edges.

Table 1. Metrics Comparison Between the Original Image and Processed Images Using Different $\beta$ Values

Edge

SNR (dB) CNR (dB) ENL Preservation

\begin{tabular}{lllll}
\hline Original & 38.67 & 2.83 & 183 & N/A \\
Median $(12 \times 12)$ & 45.72 & 3.91 & 1211 & 0.01 \\
$\beta=1.5$ & 45.64 & 3.92 & 1241 & 0.28 \\
$\beta=3$ & 39.83 & 3.61 & 1098 & 0.54 \\
$\beta=4$ & 39.71 & 3.12 & 864 & 0.68 \\
\hline
\end{tabular}

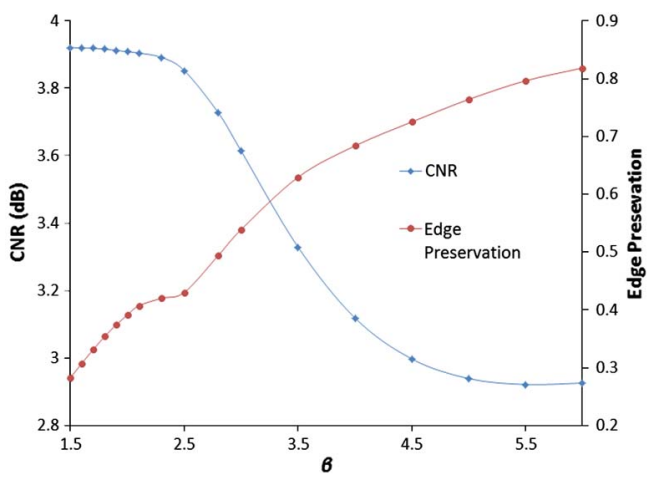

Fig. 6. (Color online) Trade-off between CNR and edge preservation by adjustment of $\beta$.

We note that one of the shortcomings is the lack of "gold standard" reference image $u$, which is not unique to our technique. Furthermore, the algorithm was run on an Intel Corei5 computer implemented in MATLAB. Speckle suppression for a $969 \times 969$ image took $\sim 7$ seconds. Future implementation in $\mathrm{C}++$ and/or GPU parallel processing may be required to perform real-time EV-OCT speckle reduction.

In summary, we proposed and demonstrated an effective speckle suppression method for EV-OCT images while preserving edge information. Furthermore, to our knowledge, we demonstrated for the first time how speckle reduction can potentially benefit EV-OCT by enhancing visibility of endovascular morphological structures with a computational method.

The work is supported by the National Engineering Research Council of Canada, the Canada Research Chairs, and Ryerson University.

\section{References}

1. A. F. Low, G. J. Tearney, B. E. Bouman, and I. Jang, Nat. Clin. Pract. Cardiovasc. Med. 3, 154 (2006).

2. A. Mariampillai, B. A. Standish, E. H. Moriyama, M. Khurana, N. R. Munce, M. K. K. Leung, J. Jiang, A. Cable, B. C. Wilson, I. A. Vitkin, and V. X. D. Yang, Opt. Lett. 33, 1530 (2008).

3. A. Mariampillai, M. K. K. Leung, M. Jarvi, B. A. Standish, K. Lee, B. C. Wilson, A. Vitkin, and V. X. D. Yang, Opt. Lett. 35, 1257 (2010).

4. M. Pircher, E. Gotzinger, R. Leitgeb, A. F. Fercher, and C. K. Hitzenberger, J. Biomed. Opt. 8, 565 (2003).

5. A. E. Desjardins, B. J. Vakoc, W. Y. Oh, S. M. R. Motaghiannezam, G. J. Tearney, and B. E. Bouma, Opt. Express 15, 6200 (2007)

6. J. W. Goodman, Speckle Phenomena in Optics (Roberts \& Company, 2007).

7. D. C. Adler, T. H. Ko, and J. G. Fujimoto, Opt. Lett. 29, 2878 (2004).

8. P. Puvanathasan and K. Bizheva, Opt. Express 15, 15747 (2007).

9. Z. Jian, Z. Yu, L. Yu, B. Rao, Z. Chen, and B. J. Tromberg, Opt. Lett. 34, 1516 (2009).

10. J. Rogowska and M. E. Brezinski, IEEE Trans. Med. Imag. 19, 1261 (2000).

11. P. Puvanathasan and K. Bizheva, Opt. Express 17, 733 (2009).

12. D. L. Marks, T. S. Ralston, and S. A. Boppart, J. Opt. Soc. Am. A 22, 2366 (2005).

13. M. Mihoko and S. Eguchi, Neural Comput. 14, 1859 (2002).

14. K. H. Y. Cheng, C. Sun, B. Vuong, K. K. C. Lee, A. Mariampillai, T. R. Marotta, J. Spears, W. J. Montanera, P. R. Herman, K. Rasmus, B. A. Standish, and V. X. D. Yang, Biomed. Opt. Express 3, 388 (2012).

15. A. Ozcan, A. Bilenca, A. E. Desjardins, B. E. Bouma, and G. J. Tearney, J. Opt. Soc. Am. A 24, 1901 (2007). 